\section{Valacyclovir Neurotoxicity and Nephrotoxicity in an Elderly Patient Complicated by Hyponatremia}

\author{
Takuya Murakami, Tetsu Akimoto, Mari Okada, Erika Hishida, \\ Taro Sugase, Atsushi Miki, Marina Kohara, Hiromichi Yoshizawa, \\ Takahiro Masuda, Takahisa Kobayashi, Osamu Saito, \\ Shigeaki Muto and Daisuke Nagata \\ Division of Nephrology, Department of Internal Medicine, Jichi Medical University, Shimotsuke, \\ Japan.
}

Drug Target Insights

Volume 12: 1-5

(C) The Author(s) 2018

Reprints and permissions:

sagepub.co.uk/journalsPermissions.nav

DOI: $10.1177 / 1177392818782899$

(S)AGE

\begin{abstract}
A 66-year-old women with no history of renal disease was admitted due to a coma and acute kidney injury with a serum creatinine level of $7.44 \mathrm{mg} / \mathrm{dL}$ which were ascribed to valacyclovir neurotoxicity and nephrotoxicity, respectively. She had received valacyclovir at a standard dosage for the treatment of herpes zoster and was finally discharged, having fully returned to her normal baseline mental status with a recovered serum creatinine level of $0.68 \mathrm{mg} / \mathrm{dL}$. We feel that awareness of this pathology remains a challenge for physicians and therefore strongly recommend the further accumulation of experiences similar to our own. Our experience underscores the pitfalls of administering valacyclovir to elderly patients who barely appear to have a favorable renal function. Several concerns regarding the therapeutic management, including blood purification strategies, that emerged in this case are also discussed.
\end{abstract}

KEYWORDS: Valacyclovir neurotoxicity, acute kidney injury, hemodialysis, hyponatremia, osmotic demyelination syndrome

RECEIVED: January 28, 2018. ACCEPTED: May 16, 2018.

TYPE: Case Report

FUNDING: The author(s) disclosed receipt of the following financial support for the research, authorship, and/or publication of this article: This study was supported in part by a

Grant-in-Aid for Research on Advanced Chronic Kidney Disease, Practical Research Project for Renal Diseases from the Japan Agency for Medical Research and Development (AMED),

and by a grant for Private University Research Branding Project from the Ministry of

Education, Science and Culture, Japan.
DECLARATION OF CONFLICTING INTERESTS: The author(s) declared no potential conflicts of interest with respect to the research, authorship, and/or publication of this article.

CORRESPONDING AUTHOR: Tetsu Akimoto, Division of Nephrology, Department of Internal Medicine, Jichi Medical University, 3311-1 Yakushiji, Shimotsuke-Shi 329-0498, Tochigi, Japan. Email: tetsu-a@jichi.ac.jp

\section{Introduction}

The administration of acyclovir antiviral therapy has been a treatment option for varicella zoster virus infection. ${ }^{1,2}$ Valacyclovir is the valyl ester of acyclovir and after its oral administration it undergoes first-pass intestinal and/or hepatic metabolism to produce active-moiety acyclovir and L-valine at a high bioavailability that is several times greater than that obtained from oral acyclovir. ${ }^{1,3}$ Both agents are well tolerated in the ordinary clinical settings; however, it is not surprising that acyclovir and oral valacyclovir share an adverse event profile that is both qualitatively and quantitatively similar. ${ }^{1}$ Subjects with chronic renal insufficiency are susceptible to adverse events characterized by neuropsychiatric manifestations. ${ }^{3,4}$ As such, dosing adjustments proportionate to renal impairment may be required. ${ }^{1,2}$ In this report, we describe our experience with a case of valacyclovir neurotoxicity accompanied by acute kidney injury (AKI) in an aged female patient who barely appeared to have acceptable renal function to receive the agent at the standard dosage for the treatment of herpes zoster $(\mathrm{HZ})$. Several management concerns that emerged in the current case are also discussed.

\section{Case Report}

A 66-year-old woman with a history of hypertension and hyperlipidemia was referred and admitted to our hospital in the middle of November 2015 due to coma accompanied by AKI. Six years earlier, she had been found to have these diseases and had been treated with atorvastatin as well as antihypertensive agents, including amlodipine, olmesartan medoxomil, and carvedilol. Although the renal parameters had not been monitored on a regular basis, her serum creatinine $(\mathrm{sCr})$ level slightly increased from $0.58 \mathrm{mg} / \mathrm{dL}$ in July 2013 to $0.69 \mathrm{mg} / \mathrm{dL}$ in September 2015, indicating declines in the estimated Cr clearance (eCrCL) determined by Cockcroft-Gault formula ${ }^{5}$ and the estimated glomerular filtration rate (eGFR) based on the revised Japanese equation ${ }^{6}$ from 72.7 to $59.5 \mathrm{~mL} / \mathrm{min}$ and 78.9 to $64.7 \mathrm{~mL} / \mathrm{min} / 1.73 \mathrm{~m}^{2}$, respectively. Eight days before this admission, she had noticed a maculopapular rash over the left ear and been empirically treated with oral cefcapene pivoxil hydrochloride $300 \mathrm{mg}$ /day combined with topical gentamicin by her general practitioner. Three days later, the rash had progressed to clusters of clear vesicles. She was then diagnosed with left trigeminal $\mathrm{HZ}$ and subjected to oral valacyclovir $1 \mathrm{~g}$ three times a day. On the fifth day of the valacyclovir treatment, the patient started to exhibit mild dysarthria characterized by slurred speech followed by a progressive deterioration of consciousness. The next day, she was brought to another emergency hospital by her son. A physical examination revealed her to be stuporous and afebrile without any facial drooping, tongue deviation, hemiparesis, or quadriparesis. Diagnostic brain computed tomography and magnetic resonance imaging revealed no remarkable abnormalities, whereas a laboratory analysis revealed the elevated levels of $\mathrm{sCr}$ of $7.44 \mathrm{mg} / \mathrm{dL}$ and 


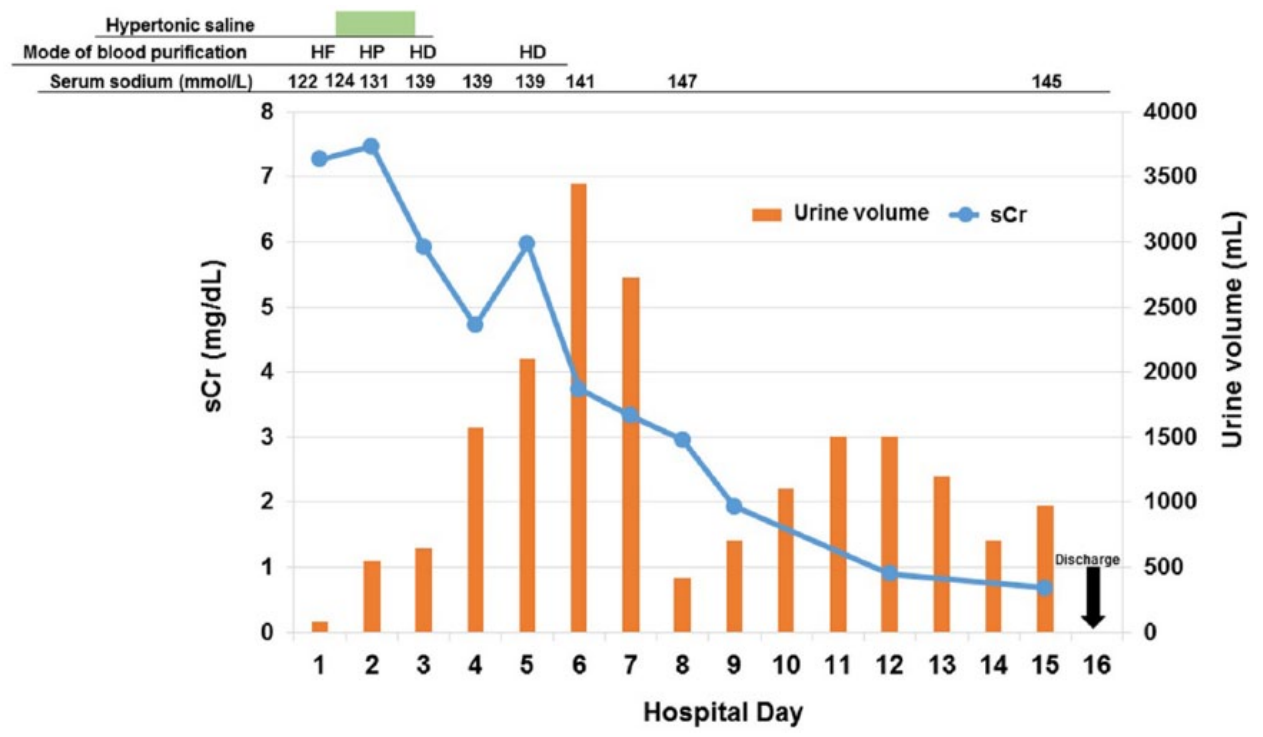

Figure 1. Serial changes in the sCr, serum sodium levels, and urine volume during the observation period. HF and HP were performed on hospital days 1 and 2, respectively, whereas the patient received the HD treatment on hospital days 3 and 5 . The administration of intravenous hypertonic saline was ceased after confirming that the patient's serum sodium level had been corrected to $139 \mathrm{mmol} / \mathrm{L}$ on hospital day 3 .

serum potassium of $6.2 \mathrm{mmol} / \mathrm{L}$. She was empirically given $10 \mathrm{mg}$ of furosemide as well as $10 \mathrm{~mL}$ of $8.5 \%$ calcium gluconate intravenously and then transferred and admitted to our hospital for further work-up.

On admission, the patient had a blood pressure of $191 / 105 \mathrm{~mm} \mathrm{Hg}$ with a pulse of 101 beats/min. She was a wellnourished woman of $152 \mathrm{~cm}$ in height and $47.4 \mathrm{~kg}$ in weight. Her consciousness level was E3V4M6 on the Glasgow Coma Scale (GCS). Renal sonography on both kidneys revealed preservation of the size with normal renal cortex echogenicity. A laboratory examination revealed the following results: hemoglobin, $11.9 \mathrm{~g} / \mathrm{dL}$; hematocrit, 36.9\%; platelet count, $15.9 \times 10^{4} / \mu \mathrm{L}$; blood urea nitrogen, $53 \mathrm{mg} / \mathrm{dL}$; sCr, $7.27 \mathrm{mg} / \mathrm{dL}$; uric acid, $8.8 \mathrm{mg} / \mathrm{dL}$; total protein, $6.4 \mathrm{~g} / \mathrm{dL}$; serum albumin, $3.3 \mathrm{~g} / \mathrm{dL}$; sodium, $122 \mathrm{mmol} / \mathrm{L}$; potassium, $5.9 \mathrm{mmol} / \mathrm{L}$; chloride, $92 \mathrm{mmol} / \mathrm{L}$; Ca, $8.3 \mathrm{mg} / \mathrm{dL}$; phosphorus, $5.0 \mathrm{mg} / \mathrm{dL}$; C3, $112 \mathrm{mg} / \mathrm{dL}$; C4, $25 \mathrm{mg} / \mathrm{dL}$; immunoglobulin (Ig) G, $1246 \mathrm{mg} /$ $\mathrm{dL}$; IgA, $239 \mathrm{mg} / \mathrm{dL}$; and IgM, $73 \mathrm{mg} / \mathrm{dL}$. A serological study revealed an increased level of C-reactive protein of $3.55 \mathrm{mg} / \mathrm{dL}$, whereas the patient was negative for antimyeloperoxidase antineutrophil cytoplasmic antibody (ANCA), antiproteinase 3-ANCA, anti-glomerular basement membrane antibodies, and anti-double-stranded DNA antibodies. No serum thyroidstimulating hormone abnormalities were found. A lumbar puncture revealed a protein concentration of $54 \mathrm{mg} / \mathrm{dL}$ and glucose concentration of $91 \mathrm{mg} / \mathrm{dL}$, whereas Gram staining failed to show microorganisms, and cultures of blood and cerebrospinal fluid (CSF) were also negative. Although we did not perform a polymerase chain reaction to screen the patient's CSF for $\mathrm{HZ}$ virus DNA, the serum and CSF acyclovir levels at this point were 26.9 and $6.25 \mu \mathrm{g} / \mathrm{mL}$, respectively; as such, valacyclovir-related pathologies were strongly suspected to be implicated in the patient's neuropsychiatric manifestations. All medications were discontinued, and she was subjected to a single session of hemofiltration (HF) for the management of anuric AKI accompanied by hyperkalemia and hyponatremia when her neurological status further worsened (E1V1M4 on GCS).

A temporary dialysis catheter was placed in the right jugular vein and a Foley catheter was inserted in the bladder to monitor the urine output. Then, HF was initiated with a high-flux hemodialyzer (FB-70U, cellulose triacetate membrane, $0.7 \mathrm{~m}^{2}$; Nipro Co., Osaka, Japan). The rates of blood flow and ultrafiltration were set at $120 \mathrm{~mL} / \mathrm{min}$ and $1000 \mathrm{~mL} / \mathrm{h}$, respectively. The HF replacement fluid was the standard formulation of Sublood ${ }^{\circledR}$-BSG (Fuso Pharmaceutical Industries Ltd., Osaka, Japan) administered after filter at $1000 \mathrm{~mL} / \mathrm{h}$. After 3 hours, the patient developed respiratory distress due to aspiration and was intubated for airway protection, and then she was placed on mechanical ventilation when an urgent laboratory analysis revealed that her serum levels of potassium and sodium were 4.1 and $124 \mathrm{mmol} / \mathrm{L}$, respectively. HF was ceased, and she was then started on intravenous 3\% hypertonic saline of varying doses to correct the reduced serum sodium level at a recommended rate according to the therapeutic guidance. ${ }^{7}$ On the following day, there was a slight improvement in the patient's neurological status (E2VTM4), and the serum sodium level increased to $131 \mathrm{mmol} / \mathrm{L}$ when the patient underwent a 3-hour session of hemoperfusion (HP) using an activated charcoal cartridge (Hemosorba CHS-350 ${ }^{\circledR}$; Asahi Medical Co., Tokyo, Japan), and it further increased to $139 \mathrm{mmol} / \mathrm{L}$ at 14 hours later. After an initial hemodialysis (HD) session, her urine output began to increase (Figure 1) along with gradual improvements in her consciousness from E3VTM6 on hospital day 3 to E4VTM6 on hospital day 5, whereas the serum levels of 
Table 1. Changes in the serum acyclovir levels just before and after the blood purification procedures.

\begin{tabular}{|c|c|c|c|}
\hline \multirow[t]{2}{*}{ HOSPITAL DAY } & \multirow{2}{*}{$\begin{array}{l}\text { MODE OF BLOOD } \\
\text { PURIFICATION }\end{array}$} & \multicolumn{2}{|c|}{ SERUM ACYCLOVIR LEVEL, $\mu \mathrm{g} / \mathrm{mL}$} \\
\hline & & BEFORE THE PROCEDURE & AFTER THE PROCEDURE \\
\hline 1 & HF & 26.9 & NA \\
\hline 2 & HP & 15.88 & NA \\
\hline 3 & HD & 4.72 & 1.95 \\
\hline 5 & $\mathrm{HD}$ & NA & 0.43 \\
\hline
\end{tabular}

Abbreviations: NA: not available.

acyclovir steadily decreased during the observation period (Table 1). The patient was eventually extubated on hospital day 7 with a favorable neurological recovery (E4V5M6), and medications including statins and antihypertensive agents were resumed thereafter. She was discharged on hospital day 16, having fully returned to her normal baseline mental status with a recovered $\mathrm{sCr}$ level of $0.68 \mathrm{mg} / \mathrm{dL}$ corresponding to an $\mathrm{eCrCL}$ of $60.4 \mathrm{~mL} / \mathrm{min}$ and $\mathrm{eGFR}$ of $65.7 \mathrm{~mL} / \mathrm{min} / 1.73 \mathrm{~m}^{2}$ At the time, the results of a urine sediment examination were unremarkable, and a urine dipstick analysis was negative for protein and occult blood.

\section{Discussion}

The clinical scenario of the present case, which was characterized by a set of conditions including neurotoxicity and AKI in an $\mathrm{HZ}$ patient receiving valacyclovir may be too common to be described in the literature. Indeed, there are numerous reports of nephrotoxic and neuropsychiatric side effects in acyclovirtreated patients, whereas AKI and various neurological manifestations are occasionally observed as characteristic phenotypes of valacyclovir toxicity. ${ }^{3,4,8-11}$ However, the significance of this report should be carefully evaluated to investigate the impact of concurrent hyponatremia on the therapeutic decisions in the present case. Furthermore, our experience underscores the pitfalls associated with valacyclovir treatment for HZ, implying its limited feasibility and safety, especially in elderly patients.

The withdrawal or discontinuation of the offending agent is the mainstay treatment for acyclovir or valacyclovir-mediated poisoning; however, there are several anecdotal reports suggesting that HD may play a role in the rapid reduction in serum acyclovir levels, thereby accelerating the recovery from renal and/or neurological disorders.4,8,11-14 Given the chemical and pharmacokinetic characteristics of acyclovir, including its small molecular weight $(225 \mathrm{Da})$, limited protein-binding rate, low steady-state volume of distribution, and high water solubility, it can be readily removed with extracorporeal procedures, including $\mathrm{HD}, \mathrm{HF}$, and hemodiafiltration, with a high elimination rate in HD. ${ }^{4,8,12,15}$ In the present case, the concurrent hyponatremia precluded us from promptly performing $\mathrm{HD}$ as the procedure to remedy volume overload, azotemia, and abnormal serum electrolyte levels results in the rapid correction of the serum sodium level and predisposes patients to a risk of osmotic demyelination syndrome (ODS). ${ }^{16}$ In this context, we decided to perform $\mathrm{HF}$, which may have a lower magnitude of sodium exchange than $\mathrm{HD},{ }^{17}$ as an initial mode of blood purification with the aim of correcting the hyperkalemia associated with AKI as well as active acyclovir removal, and it was not until the confirmation of successfully corrected serum sodium levels that HD was finally applied without any hesitation. A lack of information regarding the therapeutic benefit of $\mathrm{HF}$ in cases with valacyclovir or acyclovir toxicity ${ }^{18}$ also encouraged us to employ HP empirically as an adjunct treatment for the disease after confirming the normalization of the patient's serum potassium levels, although acyclovir is not necessarily listed as an agent that can be removed by this procedure. ${ }^{19}$ Given our experience with the current patient, we believe that HF and/or HP are viable therapeutic options in patients with hyponatremia who are at risk of developing a rate of sodium correction with HD that far exceeds the safe recommendation, although it does not allow us to objectively determine the clinical impact of each procedure on this pathology. Despite these promising outcomes, our policy regarding tailored approaches to selecting and scheduling blood purification modalities in the present patient may not be appropriate because azotemia and/or uremia may protect against the demyelination that results from the rapid correction of hyponatremia during HD. ${ }^{20-22}$ However, we believe that it is necessary to take a proactive approach before serious events become apparent. Of note, it has been reported anecdotally that uremia did not provide full protection against dialysis-related ODS. ${ }^{23}$ The reason why our patient manifested hyponatremia, which is one of the most common electrolytes disorders encountered in the ordinary clinical setting, ${ }^{7}$ may be another point that needs to be addressed carefully. Although this abnormality may occasionally be seen, ${ }^{24}$ and can arise in a wide range of diseases, a detailed medical record review, a thorough clinical interview, the patient's clinical manifestations, and the prompt and timely resolution without any recurrence during the observation period, hampered our identification of disease states other than the renal failure. ${ }^{7}$ Whether there is any association between valacyclovir toxicity and hyponatremia remains inconclusive. ${ }^{25}$ The hyponatremia may have acted as a confounder of the 
valacyclovir-mediated depression of the patient's consciousness as the clinical manifestations of hyponatremia are primarily neurological ${ }^{26}$; however, it is difficult to determine the precise contributions of each factor.

Valacyclovir or acyclovir neurotoxicity commonly develops in patients with an impaired renal function, implying a role of a declined renal clearance-mediated accumulation of acyclovir and/or its metabolites in the bloodstream. ${ }^{3,4}$ Given that the renal clearance of acyclovir absolutely exceeds that of creatinine, indicating that renal tubular secretion is actively involved in the elimination of the agent, ${ }^{1}$ it is not surprising that acyclovir can induce renal failure through precipitation within the tubular lumen, which leads to obstructive nephropathy. ${ }^{2,8}$ In rare instances, interstitial nephritis and direct tubular necrosis from acyclovir may also result in renal insufficiency. ${ }^{2,3,8}$ Alternatively, or in addition, the GFR may decline due to an acyclovir-mediated increase in the total renal vascular resistance with a reduction in the renal plasma flow. ${ }^{2,11,27}$ The reason why adverse renal events were induced in our patient remains to be clarified. The patient's prompt recovery from AKI does not support the concurrent presence of primary and/or secondary renal parenchymal damage. Instead, we believe that the diminished kidney reserve resulting from age-dependent functional and morphological changes with a moderate reduction in the GFR at baseline was likely to have played a major role, thereby making our patient vulnerable to acute stress from the agent and more likely to develop clinically relevant AKI. ${ }^{28}$ Unfavorably modulated renal hemodynamics induced by the concurrent use of angiotensin II receptor antagonists might also lay the groundwork for the development of this disease. ${ }^{11,28}$ Otherwise, our patient might have received an inappropriately high dose of valacyclovir as determining eCrCL with sCr levels measured by enzymatic but not by Jaffe assays, which are not common in Japan, without standardization can overestimate actual GFR, although the optimum strategy for solving this problem remains to be delineated. $5,29,30$

The pathogenesis of valacyclovir and/or acyclovir neurotoxicity is still a matter of debate. Although a specific therapeutic range has not been equivocally established, these values overlap with the reported trough levels, ranging from 0.5 to $3 \mu \mathrm{g} / \mathrm{mL}, 31$ whereas peak concentrations of approximately $5.6 \mu \mathrm{g} / \mathrm{mL}$ and trough levels of $<2 \mu \mathrm{g} / \mathrm{mL}$ may be efficacious and below the concentrations at which acyclovir displays apparent neurotoxicity. 1,11,32 Acyclovir slowly crosses the blood-brain barrier and is then actively transported out of the CSF compartment. ${ }^{33}$ However, the impact of the acyclovir on the pathogenesis of neurotoxicity remains to be determined because its serum levels are not necessarily above the therapeutic range in patients presenting with neuropsychiatric manifestations. ${ }^{4,34}$ Rather, we may need to focus on 9-carboxymethoxymethylguanine (CMMG), a major metabolite of acyclovir, as a candidate offender as it has been regarded as a significant reliable predictor of developing neuropsychiatric events during acyclovir treatment. ${ }^{4,35}$ Not surprisingly, the determination of serum levels of CMMG may also be a useful diagnostic tool for valacyclovir and/or acyclovir neurotoxicity, ${ }^{35}$ although such a procedure was not applicable in the current patient.

The cumulative number of publications on these topics has been steadily growing ${ }^{2-4,8-15}$; however, we are of the opinion that awareness of this pathology remains limited and that an early diagnosis remains a challenge for the physicians in the ordinary clinical settings. Needless to say, it is important to consider how to prevent the adverse events associated with valacyclovir and/or acyclovir that were described in this study. Some possible approaches include establishing a euvolemic state before the administration of the medication, slower drug infusion, dose adjustment according to the renal function, and avoiding the use of other nephrotoxic agents. ${ }^{2,36}$ The published literature lacks information on the safety of substitution with other antiviral agents; however, it has been proposed anecdotally that famciclovir may be a candidate for substitution — at least in patients who develop nephrotoxicity. ${ }^{37,38}$ However, famciclovir treatment may still be associated with some risk of neuropsychiatric events. ${ }^{39}$ Finally, we strongly recommend the accumulation of more cases similar to our own, thereby allowing us to clarify the nature of adverse valacyclovir-mediated reactions more precisely as well as establish an optimum management strategy, especially regarding appropriate valacyclovir dosing regimens in elderly patients with $\mathrm{HZ}$ who barely appear to have a favorable renal function.

\section{Author Contributions}

TMurakami and TA drafted the manuscript. MO, EH, TS, AM, MK, HY, and TMasuda made contributions to the acquisition of the clinical data. TK, OS, SM, and DN provided a detailed review of the contents and structure of the manuscript, resulting in significant changes to the original document. All authors have read and approved the final manuscript.

\section{Disclosure and Ethics}

As a requirement of publication, the authors have provided the publisher with a signed confirmation of compliance with legal and ethical obligations, including but not limited to the following: authorship and contributorship, conflicts of interest, privacy and confidentiality, and (where applicable) protection of human and animal research subjects. The authors have read and confirmed their agreement with the ICMJE authorship and conflict of interest criteria. The authors have also confirmed that this article is unique and that it is not been published nor is it under consideration by any other publication, and that they have permission from the rights holders to reproduce any copyrighted material. The external blind peer reviewers report no conflicts of interest in association with this study.

\section{REFERENCES}

1. Ormrod D, Goa K. Valaciclovir: a review of its use in the management of herpes zoster. Drugs. 2000;59:1317-1340.

2. Perazella MA. Crystal-induced acute renal failure. Am J Med. 1999;106: 459-465. 
3. Asahi T, Tsutsui M, Wakasugi M, et al. Valacyclovir neurotoxicity: clinical experience and review of the literature. Eur J Neurol. 2009;16:457-460.

4. Yang HH, Hsiao YP, Shih HC, Yang JH. Acyclovir-induced neuropsychosis successfully recovered after immediate hemodialysis in an end-stage renal disease patient. Int J Dermatol. 2007;46:883-884.

5. Cockcroft DW, Gault MH. Prediction of creatinine clearance from serum creatinine. Nephron. 1976;16(1):31-41.

6. Matsuo S, Imai E, Horio M, et al. Revised equations for estimated GFR from serum creatinine in Japan. Am J Kidney Dis. 2009;53(6):982-992.

7. Verbalis JG, Goldsmith SR, Greenberg A, Schrier RW, Sterns RH. Hyponatremia treatment guidelines 2007: expert panel recommendations. Am J Med. 2007; 120(11 Suppl 1):S1-21.

8. Krieble BF, Rudy DW, Glick MR, Clayman MD. Case report: acyclovir neurotoxicity and nephrotoxicity-the role for hemodialysis. Am J Med Sci. 1993;305:36-39.

9. Sodhi PK, Ratan SK. A case of chronic renal dysfunction following treatment with oral acyclovir. Scand J Infect Dis. 2003; 35(10):770-772.

10. Carlon R, Possamai C, Corbanese U. Acute renal failure and severe neurotoxicity following valacyclovir. Intensive Care Med. 2005; 31(11):1593.

11. Sugimoto T, Yasuda M, Sakaguchi M, et al. Oliguric acute renal failure following oral valacyclovir therapy. OJM. 2008; 101(2):164-166.

12. Leikin JB, Shicker L, Orlowski J, Blair AT, McAllister K. Hemodialysis removal of acyclovir. Vet Hum Toxicol. 1995;37:233-234.

13. Hsu CC, Lai TI, Lien WC, Chen WJ, Fang CC. Emergent hemodialysis for acyclovir toxicity. Am J Emerg Med. 2005;23:899-900.

14. Kambhampati G, Pakkivenkata U, Kazory A. Valacyclovir neurotoxicity can be effectively managed by hemodialysis. Eur J Neurol. 2011;18:e33.

15. Bleyzac N, Barou P, Massenavette B, et al. Assessment of acyclovir intraindividual pharmacokinetic variability during continuous hemofiltration, continuous hemodiafiltration, and continuous hemodialysis. Ther Drug Monit. 1999;21: 520-525.

16. Yessayan L, Yee J, Frinak S, Szamosfalvi B. Treatment of severe hyponatremia in patients with kidney failure: role of continuous venovenous hemofiltration with low-sodium replacement fluid. Am J Kidney Dis. 2014;64:305-310.

17. Di Filippo S, Manzoni C, Andrulli S, Tentori F, Locatelli F. Sodium removal during pre-dilution haemofiltration. Nephrol Dial Transplant. 2003;18:vii31-vii36.

18. Meng JB, Zheng X, Zhang G, Fang Q. Oral acyclovir induced acute renal failure. World J Emerg Med. 2011;2:310-313.

19. Winchester JF, Harboud NB, Charen E, Ghannoum M. Use of dialysis and hemoperfusion in the treatment of poisoning. In: Daugirdas JT, Blake PG, Ing TS, eds. Handbook of Dialysis, 5th ed. Philadelphia, PA: Wolters Kluwer; 2015:368-390.

20. Oo TN, Smith CL, Swan SK. Does uremia protect against the demyelination associated with correction of hyponatremia during hemodialysis? a case report and literature review. Semin Dial. 2003;16:68-71.

21. Sirota JC, Berl T. Is osmotic demyelination a concern dialyzing hyponatremic patients? Semin Dial. 2011;24:407-409.
22. Dhrolia MF, Akhtar SF, Ahmed E, Naqvi A, Rizvi A. Azotemia protects the brain from osmotic demyelination on rapid correction of hyponatremia. Saudi J Kidney Dis Transpl. 2014;25:558-566.

23. Huang WY, Weng WC, Peng TI, Ro LS, Yang CW, Chen KH. Central pontine and extrapontine myelinolysis after rapid correction of hyponatremia by hemodialysis in a uremic patient. Ren Fail. 2007;29:635-638.

24. Ferreira M, Vega C, Rivas B, Selgas R. Acute renal failure and severe neurotoxicity after unintentional overdose of valacyclovir in a geriatric population: a case report. Nefrología. 2018;38:323-325.

25. Cortejoso L, Gómez-Antúnez M, Muiño-Míguez A, Durán-García ME, Sanjurjo-Sáez M. Acyclovir and hyponatremia: a case report. Am J Ther. 2014;21:e151-e153.

26. Sterns RH. Disorders of plasma sodium-causes, consequences, and correction. NEngl J Med. 2015;372:55-65.

27. Dos Santos Mde F, Dos Santos OF, Boim MA, et al. Nephrotoxicity of acyclovir and ganciclovir in rats: evaluation of glomerular hemodynamics. $J$ Am Soc Nephrol. 1997;8:361-367.

28. Coca SG. Acute kidney injury in elderly persons. Am J Kidney Dis. 2010;56: 122-131.

29. Ando Y, Minami H, Saka H, Ando M, Sakai S, Shimokata K. Adjustment of creatinine clearance improves accuracy of Calvert's formula for carboplatin dosing. BrJ Cancer. 1997;76:1067-1071.

30. Delanaye P, Cavalier E, Pottel H. Serum creatinine: not so simple! Nephron. 2017;136:302-308.

31. Adair JC, Gold M, Bond RE. Acyclovir neurotoxicity: clinical experience and review of the literature. South Med J. 1994;87:1227-1231.

32. Collins A, Krieff D, Smith C, Singer C. Neuropsychiatric toxicity in a patient undergoing hemodialysis and receiving treatment with oral acyclovir. Clin Infect Dis. 1996;22:187-188.

33. Pouplin T, Pouplin JN, Van Toi P, et al. Valacyclovir for herpes simplex encephalitis. Antimicrob Agents Chemother. 2011;55:3624-3626.

34. de Knegt RJ, van der Pijl H, van Es LA. Acyclovir-associated encephalopathy, lack of relationship between acyclovir levels and symptoms. Nephrol Dial Transplant. 1995;10:1775-1777.

35. Helldén A, Odar-Cederlöf I, Diener P, et al. High serum concentrations of the acyclovir main metabolite 9-carboxymethoxymethylguanine in renal failure patients with acyclovir-related neuropsychiatric side effects: an observational study. Nephrol Dial Transplant. 2003;18:1135-1141.

36. Fleischer R, Johnson M. Acyclovir nephrotoxicity: a case report highlighting the importance of prevention, detection, and treatment of acyclovir-induced nephropathy. Case Rep Med. 2010;2010:602783.

37. Htwe TH, Bergman S, Koirala J. Famciclovir substitution for patients with acyclovir-associated renal toxicity. J Infect. 2008;57:266-268.

38. Yoon H, Rhew KY. Famciclovir as an antiviral agent for a patient with acute renal failure. Int J Clin Pharm. 2013;35:173-175.

39. Gales BJ, Gales MA. Confusion and bradykinesia associated with famciclovir therapy for herpes zoster. Am J Health Syst Pharm. 1996;53:1454, 1456. 